\title{
Unique Diacidic Fragments Inhibit the OXA-48 \\ Carbapenemase and Enhance the Killing of E. coli Producing OXA-48
}

Doris Mia Taylor ${ }^{1}$, Justin Anglin²,3, Liya Hư ${ }^{1}$, Lingfei Wang ${ }^{4}$, Banumathi Sankaran ${ }^{5}$, Jin Wang ${ }^{4}$, Martin M. Matzuk ${ }^{2,3,4}$, B.V. Venkataram Prasad ${ }^{1}$ and Timothy Palzkill ${ }^{1,4 *}$

${ }^{1}$ Verna and Marrs McLean Department of Biochemistry and Molecular Biology, Baylor College of Medicine, Houston, TX, 77030, USA

${ }^{2}$ Center for Drug Discovery, Baylor College of Medicine, Houston, TX, 77030 USA

${ }^{3}$ Department of Pathology \& Immunology, Baylor College of Medicine, Houston, TX, 77030, USA

${ }^{4}$ Department of Pharmacology and Chemical Biology, Baylor College of Medicine, Houston, TX, 77030, USA

${ }^{5}$ Berkeley Center for Structural Biology, Advanced Light Source, Lawrence Berkeley National Laboratory, CA, 94720, USA

*Corresponding Author: Timothy Palzkill, E-mail: timothyp@bcm.edu

\section{Quality Control of Compounds}

All compounds were purchased from commercial vendors and assessed for quality and purity using ${ }^{1} \mathrm{H}$ NMR and HRMS. ${ }^{1} \mathrm{H}$ NMR spectra were recorded on a $400 \mathrm{MHz}$ Varian NMR spectrometer. Chemical shifts $(\delta)$ were given in ppm with reference to solvent signals $\left[{ }^{1} \mathrm{H} N M R\right.$ : DMSO-d6 (2.50), $\mathrm{D}_{2} \mathrm{O}$ (4.79)]. HRMS was measured on a Thermo Scientific Orbitrap Fusion Lumos. The compounds tested were purchased from the following vendors:

1) Cmpd 2.1 (Chem-Implex Int'I Inc., Wood Dale, IL)

2) Cmpd 2.2 (Combi-Blocks, San Diego, CA)

3) Cmpd 2.3 (Combi-Blocks, San Diego, CA)

4) Cmpd 3.1 (Tokyo Chemical Industry America, Portland, OR))

5) Cmpd 3.2 (Sigma Aldrich, St. Louis, MO)

6) Cmpd 3.3 (EMD Millipore Corporation, Billerica, MA)

7) Cmpd 3.4 (Apollo Scientific, Stockport, UK)

8) Cmpd 3.5 (Synthonix Inc., Wake Forest, NC)

9) Cmpd 4.1 (Sigma Aldrich, St. Louis, MO)

10) Cmpd 4.2 (Sigma Aldrich, St. Louis, MO)

11) Cmpd 4.3(Sigma Aldrich, St. Louis, MO)

12) Cmpd 4.4 (Combi-Blocks, San Diego, CA)

13) Cmpd 5.1 (Sigma Aldrich, St. Louis, MO)

14) Cmpd 5.2 (Sigma Aldrich, St. Louis, MO)

15) Cmpd 6.1 (Sigma Aldrich, St. Louis, MO) 

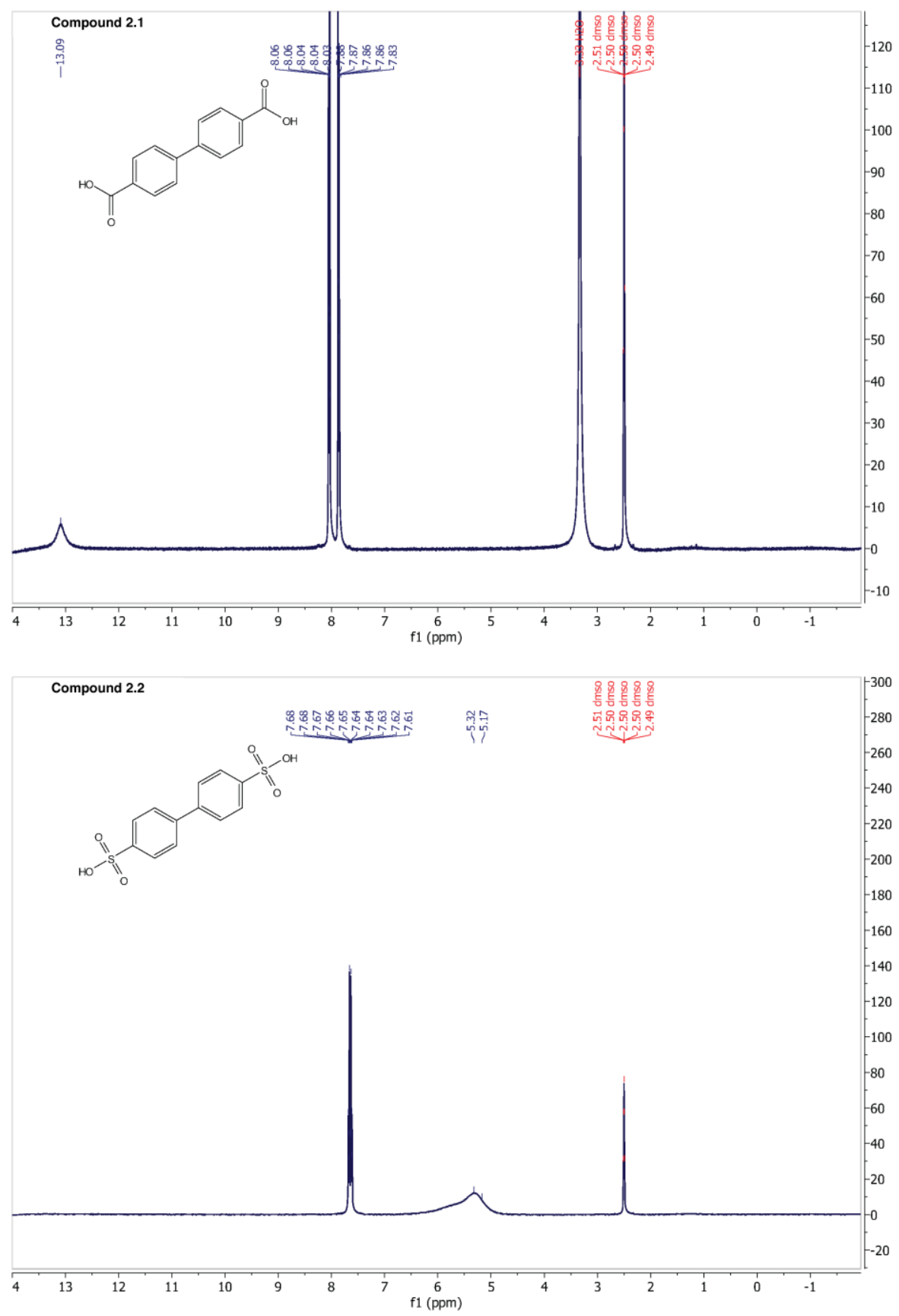

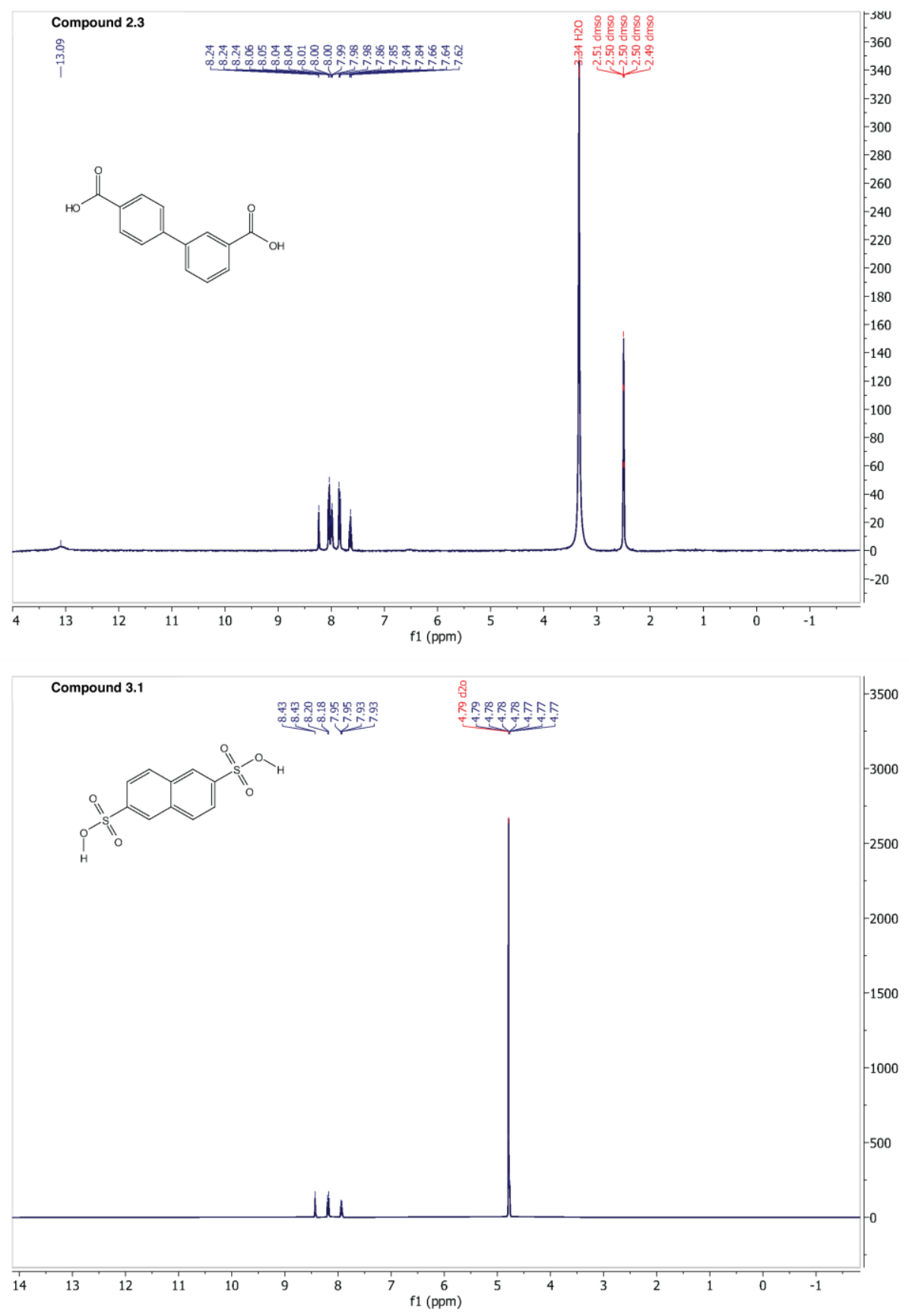

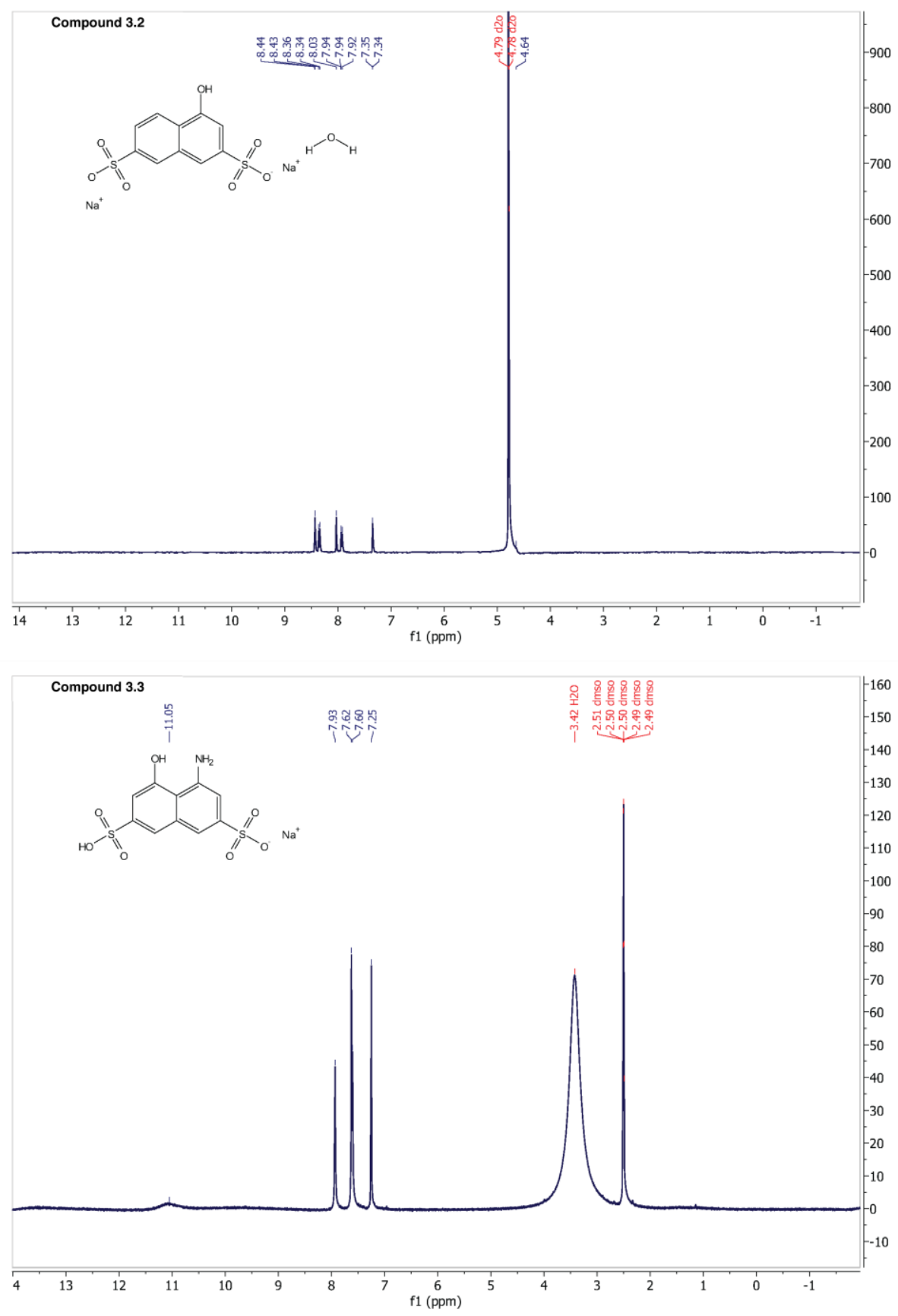

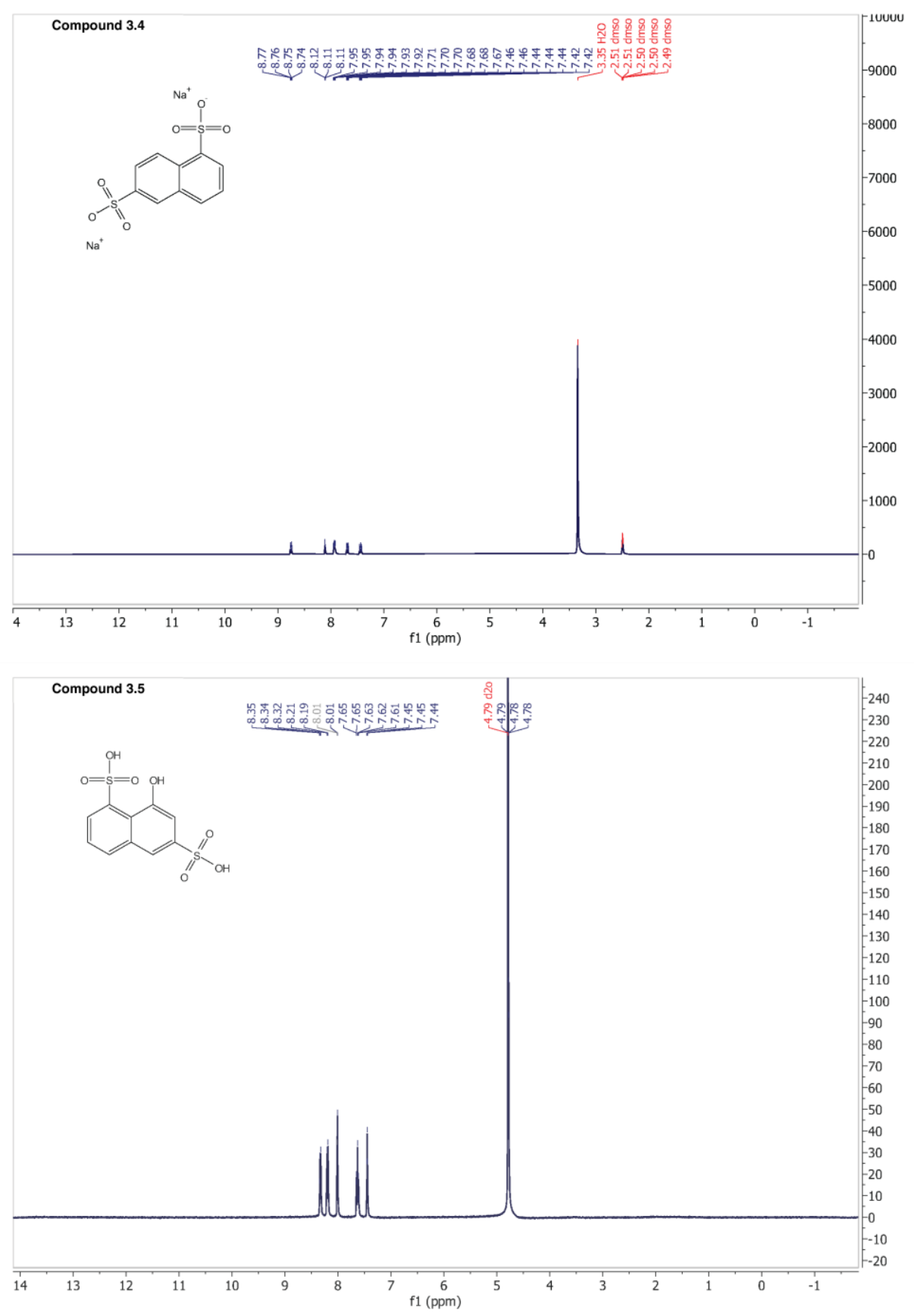

S5 

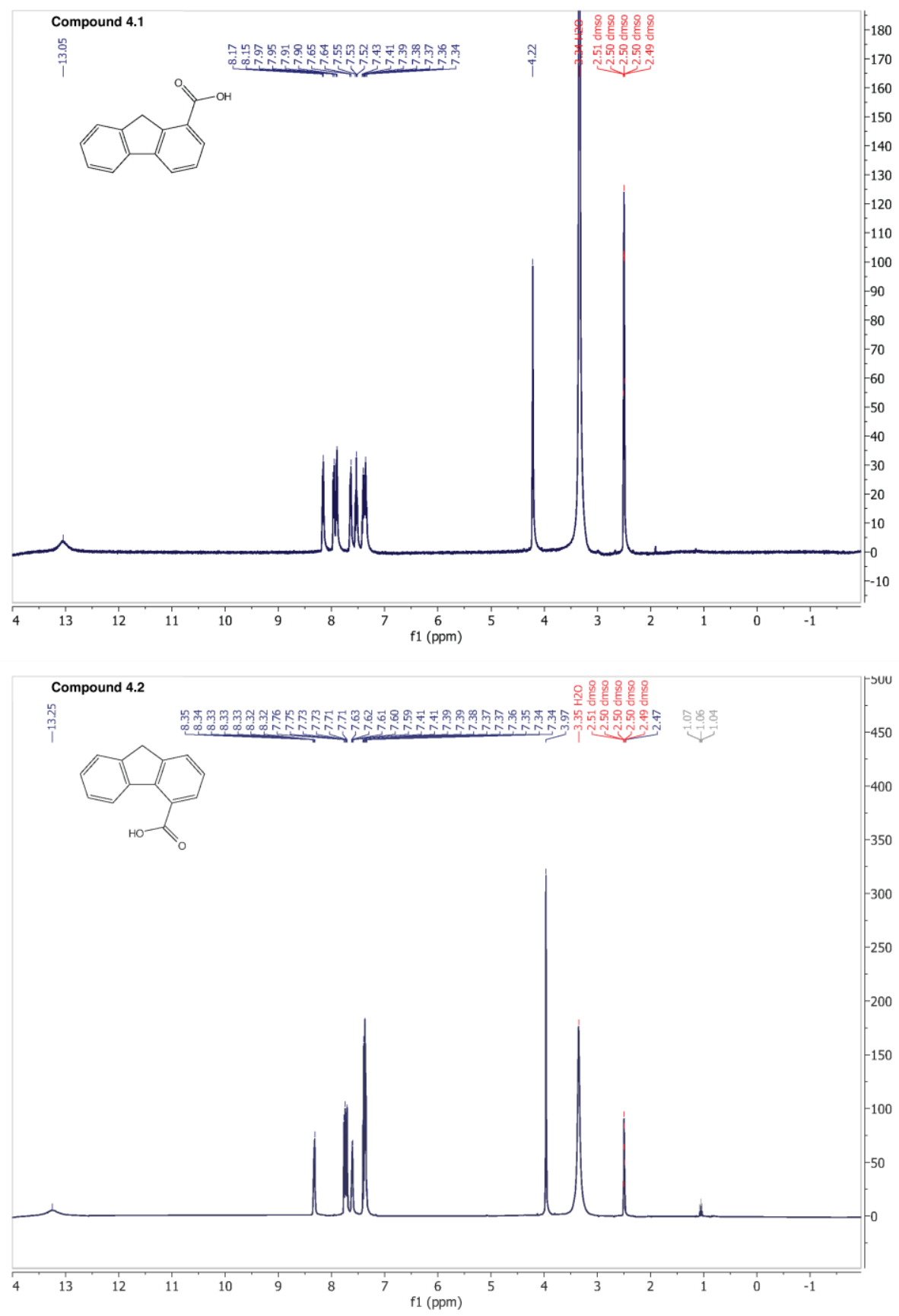

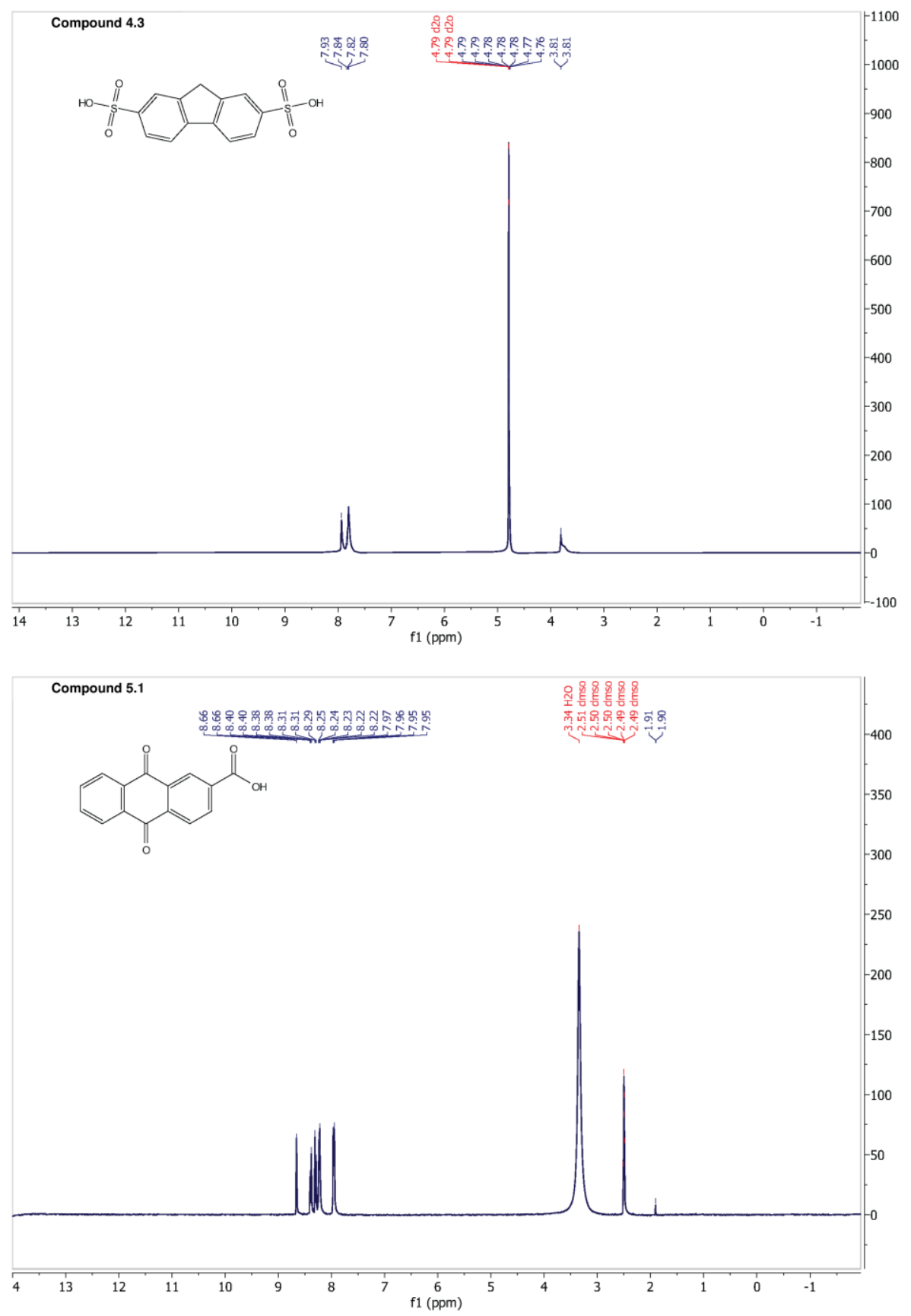

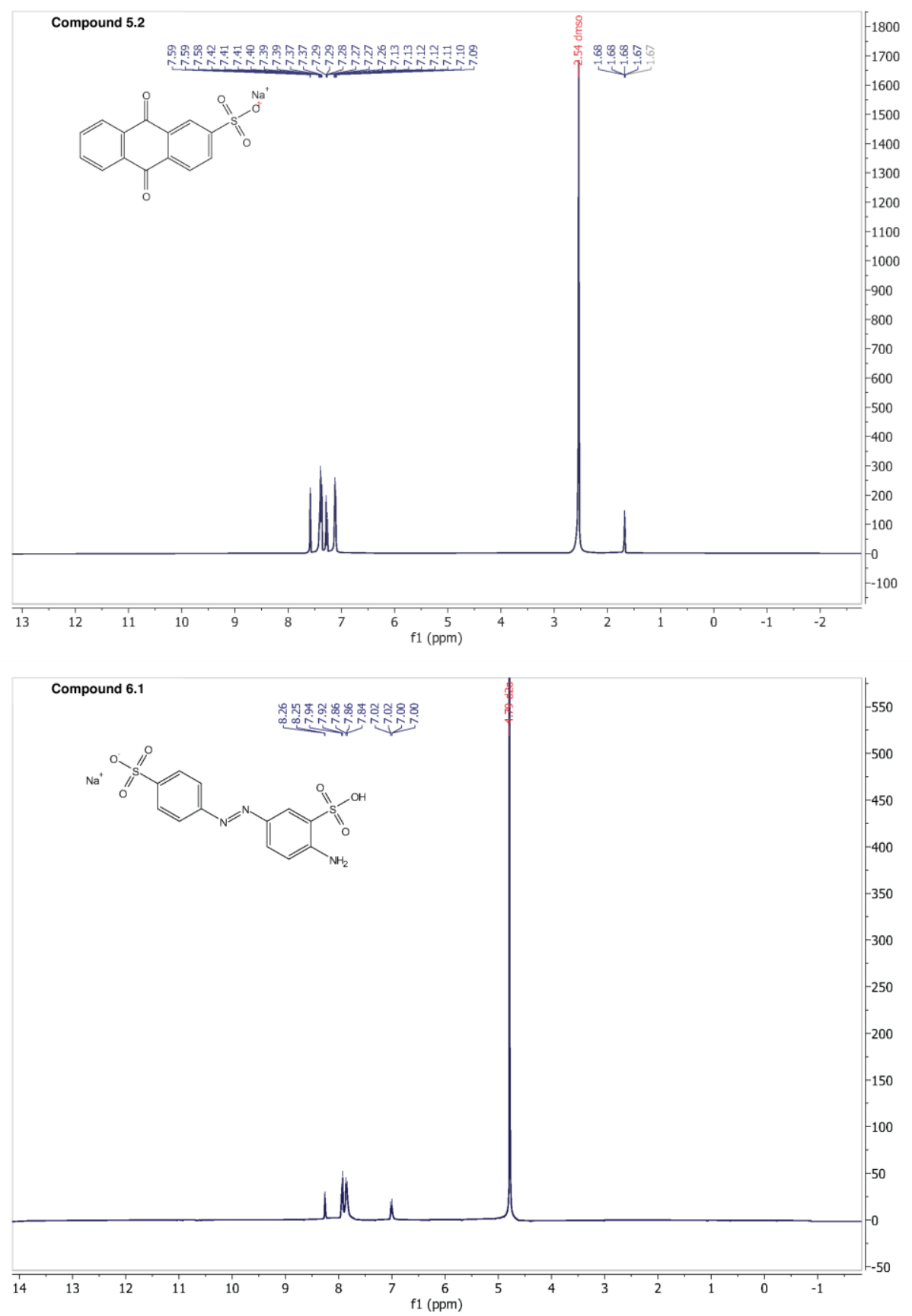

Figure S1. Series of H NMR spectra of the compounds used in this study. The expected $\mathrm{m} / \mathrm{z}$ values are shown above the peaks. 
Table S1. Mass charge ratios $(\mathrm{m} / \mathrm{z}$ ) observed via HRMS

\begin{tabular}{|c|c|c|c|}
\hline Compound I.D. & m/z Expected & $\mathrm{m} / \mathrm{z}$ Observed & Ion Type \\
\hline 2.1 & \multirow{5}{*}{$\begin{array}{l}241.05008 \\
155.98811 \\
243.04058 \\
241.05008 \\
142.98029\end{array}$} & 241.05316 & {$[\mathrm{M}-\mathrm{H}]-$} \\
\hline 2.2 & & 155.9897 & {$[\mathrm{M}-2 \mathrm{H}] 2-$} \\
\hline 2.3 & & 243.04309 & {$[\mathrm{M}-\mathrm{H}]-$} \\
\hline 2.4 & & 241.05272 & {$[\mathrm{M}-\mathrm{H}]-$} \\
\hline 3.1 & & 142.981 & {$[\mathrm{M}-2 \mathrm{H}] 2-$} \\
\hline 3.2 & 150.977745 & 150.97942 & [M-2Na]2- \\
\hline 3.3 & 158.483195 & 158.48381 & {$[\mathrm{M}-\mathrm{H}-\mathrm{Na}] 2-$} \\
\hline 3.4 & 142.98029 & 142.98094 & {$[\mathrm{M}-2 \mathrm{Na}] 2-$} \\
\hline 3.5 & 150.977745 & 150.97826 & {$[\mathrm{M}-2 \mathrm{H}] 2-$} \\
\hline 4.1 & \multirow{2}{*}{$\begin{array}{l}209.06025 \\
209.06025 \\
\end{array}$} & 209.06047 & {$[\mathrm{M}-\mathrm{H}]-$} \\
\hline 4.2 & & 209.06065 & {$[\mathrm{M}-\mathrm{H}]-$} \\
\hline 4.3 & 161.988117 & 161.98865 & {$[\mathrm{M}-2 \mathrm{Na}] 2-$} \\
\hline 5.1 & \multirow{2}{*}{$\begin{array}{l}251.03443 \\
287.00142 \\
\end{array}$} & 251.03534 & {$[\mathrm{M}-\mathrm{H}]-$} \\
\hline 5.2 & & 287.00189 & [M-H]- \\
\hline 6.1 & 177.49664 & 177.49713 & {$[\mathrm{M}-2 \mathrm{H}] 2-$} \\
\hline
\end{tabular}




\section{Additional Structural Insights}
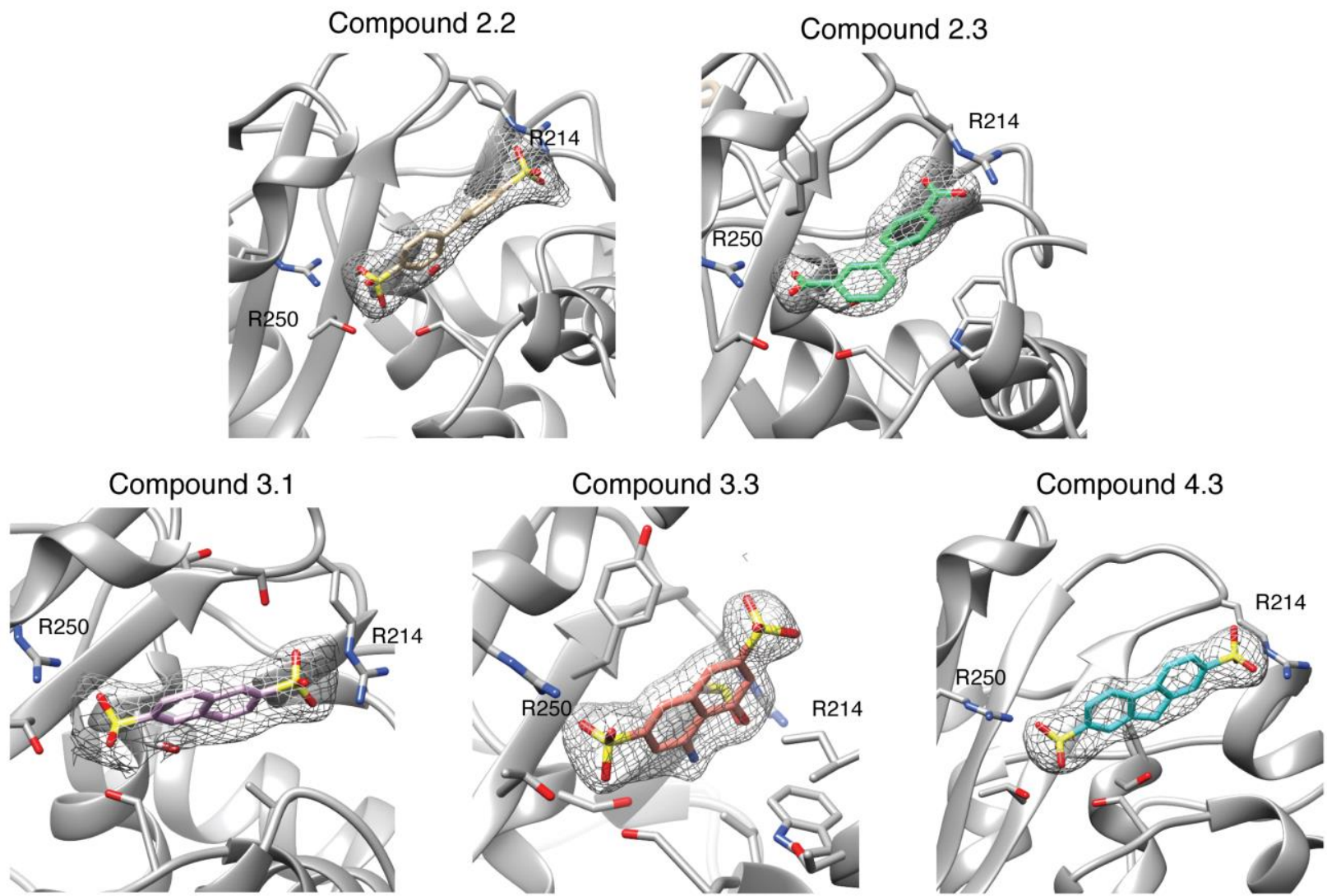

Figure S2. Polder OMIT were created using PHENIX to highlight the ligand density of the various compounds co-crystallized with OXA-48. ${ }^{1}$ Residues Arg214 and Arg250 are denoted to convey the orientation. 
Table S2. X-ray crystallography statistics of ligand fit to experimental data for OXA-48 costructures.

\begin{tabular}{|c|c|c|c|c|}
\hline Ligand & $\begin{array}{c}\text { Associated } \\
\text { OXA-48 } \\
\text { Chain }\end{array}$ & $\mathrm{RSCC}_{1}$ & $\mathbf{R S R}_{\mathbf{2}}$ & $Q<0.9_{3}$ \\
\hline \multirow{2}{*}{2.2 (VBV) } & $A$ & 0.95 & 0.11 & 0 \\
\hline & B & 0.95 & 0.10 & 20 \\
\hline \multirow{4}{*}{2.3 (VAJ) } & $A$ & 0.97 & 0.11 & 0 \\
\hline & B & 0.98 & 0.10 & 0 \\
\hline & $\mathrm{C}$ & 0.97 & 0.09 & 0 \\
\hline & D & 0.98 & 0.11 & 0 \\
\hline \multirow{2}{*}{3.1 (BIH) } & A & 0.94 & 0.15 & 0 \\
\hline & B & 0.95 & 0.14 & 0 \\
\hline \multirow{2}{*}{$3.3(310)$} & $A$ & 0.94 & 0.12 & 0 \\
\hline & B & 0.92 & 0.14 & 0 \\
\hline \multirow{4}{*}{$4.3(931)$} & $A$ & 0.97 & 0.14 & 0 \\
\hline & B & 0.98 & 0.15 & 0 \\
\hline & $C$ & 0.96 & 0.19 & 0 \\
\hline & D & 0.97 & 0.18 & 0 \\
\hline
\end{tabular}

${ }^{1}$ Real space correlation coefficient

2 Real space R-value

${ }^{3}$ Number of atoms with an occupancy less than 0.9

\section{References}

(1) Liebschner, D.; Afonine, P. V.; Moriarty, N. W.; Poon, B. K.; Sobolev, O. V.; Terwilliger, T. C.; Adams, P. D. Polder Maps: Improving OMIT Maps by Excluding Bulk Solvent. Acta Crystallogr Sect D Struct Biology 2017, 73 (Pt 2), 148-157. https://doi.org/10.1107/s2059798316018210. 